\title{
Variations dans le temps des populations d'Helminthes parasites de Rana temporaria $L$.
}

\author{
par Clande COMBES, Nicole LEGER et Bernard PESSON \\ (Collaboration technique M.-Th. Alméras) \\ Centre Universitaire, Avenue de Villeneuve, F. 66 -Perpignan \\ Faculté de Pharmacie, 4, avenue de l'Observatoire, F. 75 - Paris, 6 e
}

\begin{abstract}
Résumé
Une enquête a été menée dans six localités particulièrement représentatives du parasitisme de Rana temporaria $\mathbf{L}$. dans l'Est des Pyrénées. La confrontation des résultats obtenus en 1970 et en 1964-1966 (Combes) met en évidence une remarquable stabilité qualitative du parasitisme de Rana temporaria dans ces six stations.
\end{abstract}

\section{Summary}

An investigation has been conducted in six localities which specially represent the parasitism of Rana temporaria L. in the Eastern Pyrenees. Comparison of results achieved in 1970 and 1964-1966 (Combes) shows a remarquable qualitative stability of the parasitism of Rana temporaria in these six localities.

L'un de nous, dans un précédent travail (1968), a étudié l'épidémiologie de Digènes et de Monogènes d'Amphibiens dans l'Est des Pyrénées. Il nous a paru intéressant de rechercher dans quelle mesure certaines données de ce travail restaient valables au bout de plusieurs années. 
Notre étude portera sur les parasites de Rana temporaria L. dont nous résumons les caractéristiques dans ie tableau suivant:

\begin{tabular}{|c|c|c|c|}
\hline TAXONOMIE & PARASITES & $\begin{array}{c}\text { STADE PRÉSENT } \\
\text { CHEZ } R \text {. } \\
\text { temporaria }\end{array}$ & НАBITAT \\
\hline $\begin{array}{l}\text { Monogènes } \\
\text { Nématodes }\end{array}$ & $\begin{array}{l}\text { Haematoloechus pyrenaicus, Combes, } \\
\quad 1965 . \\
\text { Haplometra cylindracea (Zeder, 1800). } \\
\text { Opisthioglyphe rastellus (Olsson, 1876). } \\
\text { Gorgodera euzeti, Lees et Combes, 1968. } \\
\text { Gorgoderina vitelliloba (Olsson, 1876). } \\
\text { Euryhelmis squamula (Rudolphi, 1819). } \\
\text { Polystoma integerrimum (Fröhlich, 1798). } \\
\text { Rhabdias bufonis (Schrank, 1788). } \\
\text { Cosmocerca ornata (Dujardin, 1845). } \\
\text { Oswaldocruzia filiformis (Goeze, 1782). }\end{array}$ & $\begin{array}{c}\text { adulte } \\
\ll \\
\ll \\
\text { métacercaire } \\
\text { adulte } \\
\varangle\end{array}$ & $\begin{array}{l}\text { poumon } \\
\text { poumon } \\
\text { tube digestif } \\
\text { vessie } \\
\text { vessie } \\
\text { peau } \\
\text { vessie } \\
\text { poumon } \\
\text { tube digestif } \\
\text { tube digestif }\end{array}$ \\
\hline
\end{tabular}

\section{Description des milieux étudiés.}

L'enquête est faite dans une région d'altitude élevée recouvrant la Cerdagne et une partie de l'Andorre (Est des Pyrénées).

Parmi les localités décrites dans le travail de 1968, nous en avons sélectionné six qui nous ont paru représentatives des différents aspects du parasitisme de Rana temporaria dans cette région.

Nous avons représenté (fig. i) le plan de situation de ces stations et (fig. 2) la carte détaillée de chacune d'elles. Nous en donnerons une description sommaire.

— Localité 1: Bésines (Altitude : $1.990 \mathrm{~m}$ ).

Dans le secteur prospecté, le torrent des Bésines traverse une zone peu inclinée, correspondant vraisemblablement à un ancien étang atterri; il y forme de nombreux méandres entre lesquels se trouvent des bras morts et des pozzines. On est à la limite entre les étages subalpin et alpin et le cours d'eau chemine à travers des pelouses. 


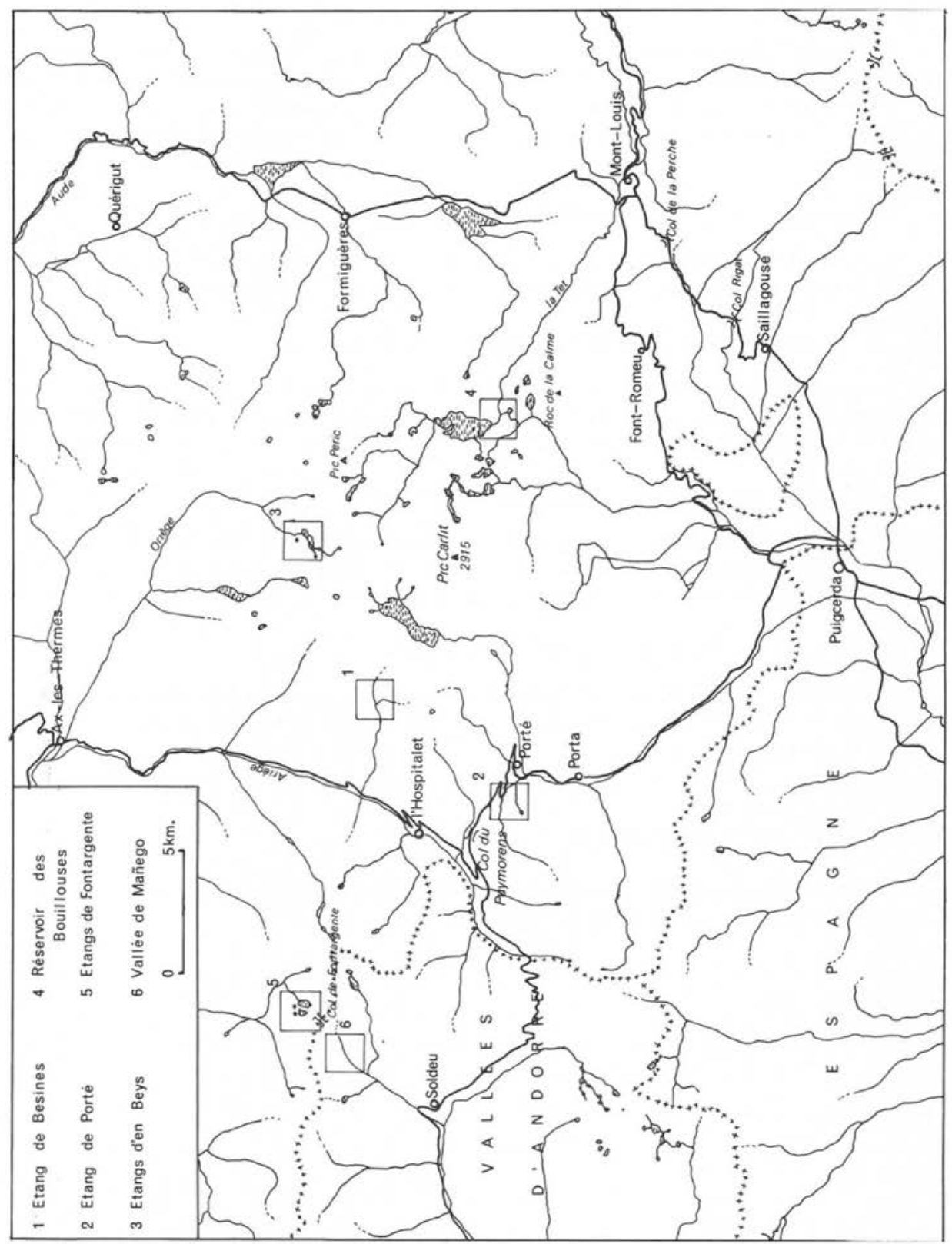

Fig. 1 
— Localité 2: Porté (Altitude : $2.040 \mathrm{~m}$ ).

Les captures ont été effectuées sur les bords de l'étang de Porté, situé dans une cuvette surélevée au-dessus de la vallée du Carol. Cet étang est de dimensions modestes (environ 200 sur $300 \mathrm{~m}$ ), peu profond et riche en végétation (Ményanthes notamment). Il est à la limite supérieure de l'étage subalpin et bordé de pelouses et de landes à rhododendrons semées de quelques pins.

— Localité 3: En Beys (Altitude : $1.960 \mathrm{~m}$ ).

Il s'agit d'un ensemble de plusieurs étangs reliés par des cours d'eau torrentueux, situés comme le précédent à la limite supérieure de l'étage subalpin, dans un paysage très dénudé.

— Localité 4: Les Bouillouses (Altitude : $2.000 \mathrm{~m}$ ).

Les grenouilles proviennent du marécage à carex situé en aval du barrage, devant le chalet-laboratoire de l'Université de Paris. La forêt de pins à crochets (limite supérieure de l'étage montagnard) est très proche.

— Localité 5: Fontargente (Altitude : $2.150 \mathrm{~m}$ ).

Nous avons prospecté le plus grand des trois étangs de Fontargente, très pauvre en végétation, et son déversoir. Le paysage, pelouses coupées de quelques buissons et rhododendrons, est caractéristique du sommet de l'étage subalpin.

— Localité 6: Mañego (Altitude : $2.100 \mathrm{~m}$ ).

Les captures ont été effectuées le long du torrent de Mañego. Comme la station précédente, dont elle n'est séparée que par un col assez bas, celle-ci se situe un peu au-dessous de l'étage des pelouses alpines.

\section{Mode d'expression des résultats.}

Dans le tableau suivant, nous résumons l'ensemble des autopsies pratiquées de 1964 à 1966 d'une part, et en 1970 d'autre part (1).

\begin{tabular}{|c|c|c|c|c|c|c|c|c|c|c|c|c|}
\hline Stations & \multicolumn{2}{|c|}{1} & \multicolumn{2}{|c|}{2} & \multicolumn{2}{|c|}{3} & \multicolumn{2}{|c|}{4} & \multicolumn{2}{|c|}{5} & \multicolumn{2}{|c|}{6} \\
\hline & $0^{*}$ & $q$ & $\sigma^{*}$ & $q$ & $0^{*}$ & $q$ & $\sigma^{*}$ & $q$ & के & $q$ & के & $q$ \\
\hline $1964-1966 \quad \ldots$ & 44 & 19 & 40 & 4 & 17 & 15 & 29 & 8 & 23 & 17 & 13 & 18 \\
\hline $1970 \ldots$ & 37 & 24 & 61 & 1 & 37 & 19 & 53 & 15 & 32 & 31 & 28 & 24 \\
\hline
\end{tabular}

(1) On notera que presque toujours le nombre de mâles capturés est supérieur à celui des femelles. Cette sex ratio estivale a été expliquée par Combes (1968). 
Pour chaque Helminthe, nous avons calculé dans chaque station :

1) La fréquence du parasitisme (nombre d'hôtes parasités pour cent). Celle-ci est exprimée de deux manières :

a) pour chaque Helminthe, un graphique (fig. 3-4) représente la fréquence de l'infestation dans chacune des six stations, en 1964-1965 (tirets) et en 1970 (trait plein) ;

b) pour chaque station, nous avons figuré les polygones de fréquence des Helminthes (fig. 5, 6, 7, 8), en 1964-1965 (blanc) et 1970 (sombre).

2) Pour les Trématodes, le rapport de la population du parasite à la population de l'hôte (nombre de parasites pour cent hôtes) (fig. 5, 6, 7).

Ce rapport est exprimé sous la forme de polygones permettant la comparaison, comme précédemment, entre 1964-1965 (pointillés) et 1970 (hachures), l'échelle est logarithmique (1).

\section{Interprétation des résultats.}

Dans les stations de Bésines et Porté qui se caractérisent par la présence de Polystomes associés à des Gorgoderidae et Haplometra cylindracea sans Opisthioglyphe rastellus, les données qualitatives de l'infestation restent les mêmes; sur le plan quantitatif, on note un augmentation générale du parasitisme à Bésines, tandis qu'à Porté, on observe des variations dans les deux sens. On notera que si la première de ces stations ne nous a pas paru avoir évolué au cours des cinq dernières années, la deuxième a été profondément remaniée par l'installation d'un petit barrage qui a provoqué l'élévation du niveau et par là même un changement dans la nature des berges.

Dans les stations des Bouillouses et En Beys, caractérisées par la présence de Opisthioglyphe rastellus, associé à des Gorgoderidae, Haplometra cylindracea et un très faible effectif de Polystomes, les données qualitatives n'ont pas davantage varié. Dans les deux localités, on note un accroissement des Opisthioglyphe rastellus. A En Beys, il y a tendance à un léger accroissement général des fréquences; aux Bouillouses, station perturbée par l'envahissement touristique, les variations sont plus capricieuses.

(1) Les chiffres donnés pour 1964-1965 à propos des Digènes et Monogènes ont les origines suivantes: pour les stations $1,3,4,5,6$, ils sont extraits des statistiques générales publiées par Combes (1968); pour la station 2, ils concernent un prélèvement particulier réalisé en juillet 1965; les chiffres donnés pour 1964-1965 à propos des Nématodes résultent des enquêtes réalisées dans les conditions exactes de celles qui précèdent, mais non publiées jusqu'ici. 
A Fontargente, caractérisée par la présence d'Opisthioglyphe rastellus, associée à des Gorgoderidae et Haplometra cylindracea, et l'absence rigoureuse de Polystomes, les données qualitatives initiales restent là aussi valables. Quantitativement, il y a augmentation ou stabilité du parasitisme.

A Mañego enfin, station initialement caractérisée par la présence de Gorgoderidae, associés à Haplometra cylindracea, et l'absence de Polystomes et d'Opisthioglyphe rastellus (1), s'observent les seules variations qualitatives de notre enquête : l'une concerne l'apparition de Polystoma integerrimum, qui reste toutefois très discrète, mais cette station est située en bordure de la «zone à Polystomes » (Combes, 1968, cartes n ${ }^{\text {os }} 6$ et 7) puisqu'il s'agit d'un torrent se jetant dans un cours d'eau où ce Monogène est courant, les Grenouilles pouvant se déplacer de l'un à l'autre ; la seconde concerne l'apparition de Rhabdias bufonis pour lequel la même explication est valable, ce Nématode parasitant un tiers environ des Grenouilles de la vallée de la Valira voisine.

En conclusion, on peut dire que qualitativement il existe sur un intervalle de cinq années, une stabilité remarquable du parasitisme de Rana temporaria dans nos six loca-

(1) Les infestations signalées en 1964-1965 ne sont pas décomptées ici, en raison de leur caractère indiscutablement accidentel (le cycle ne peut, faute de mollusques nécessaires, se dérouler dans cette station et ces infestations sont dues à des déplacements d'insectes infestés).
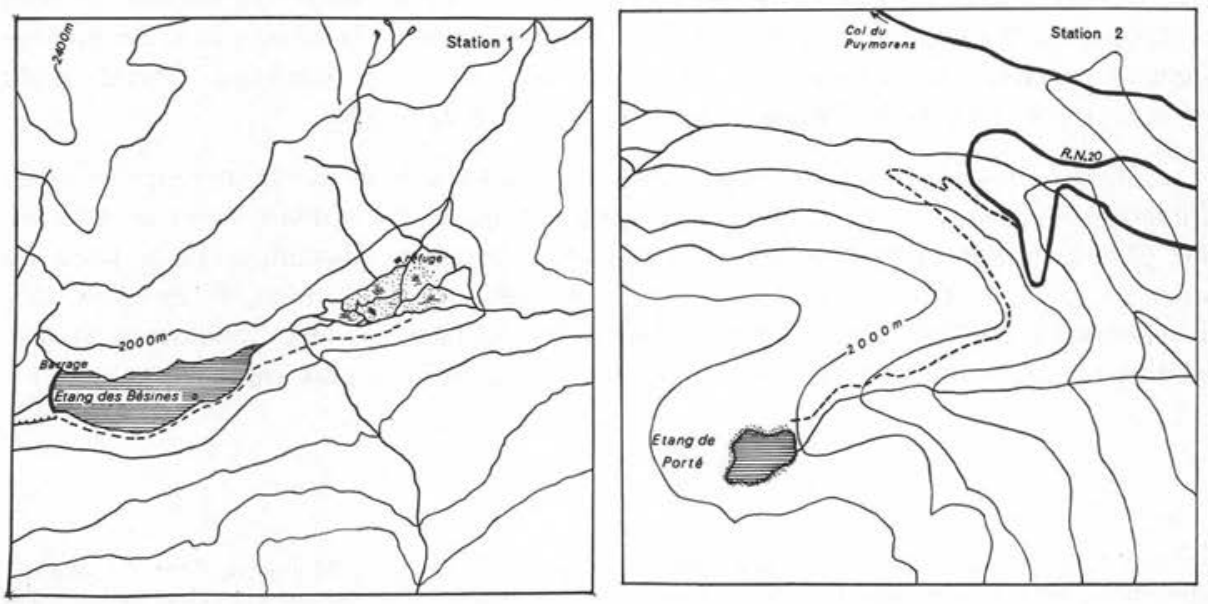

FIG. 2. - (Voir légende ci-contre) 

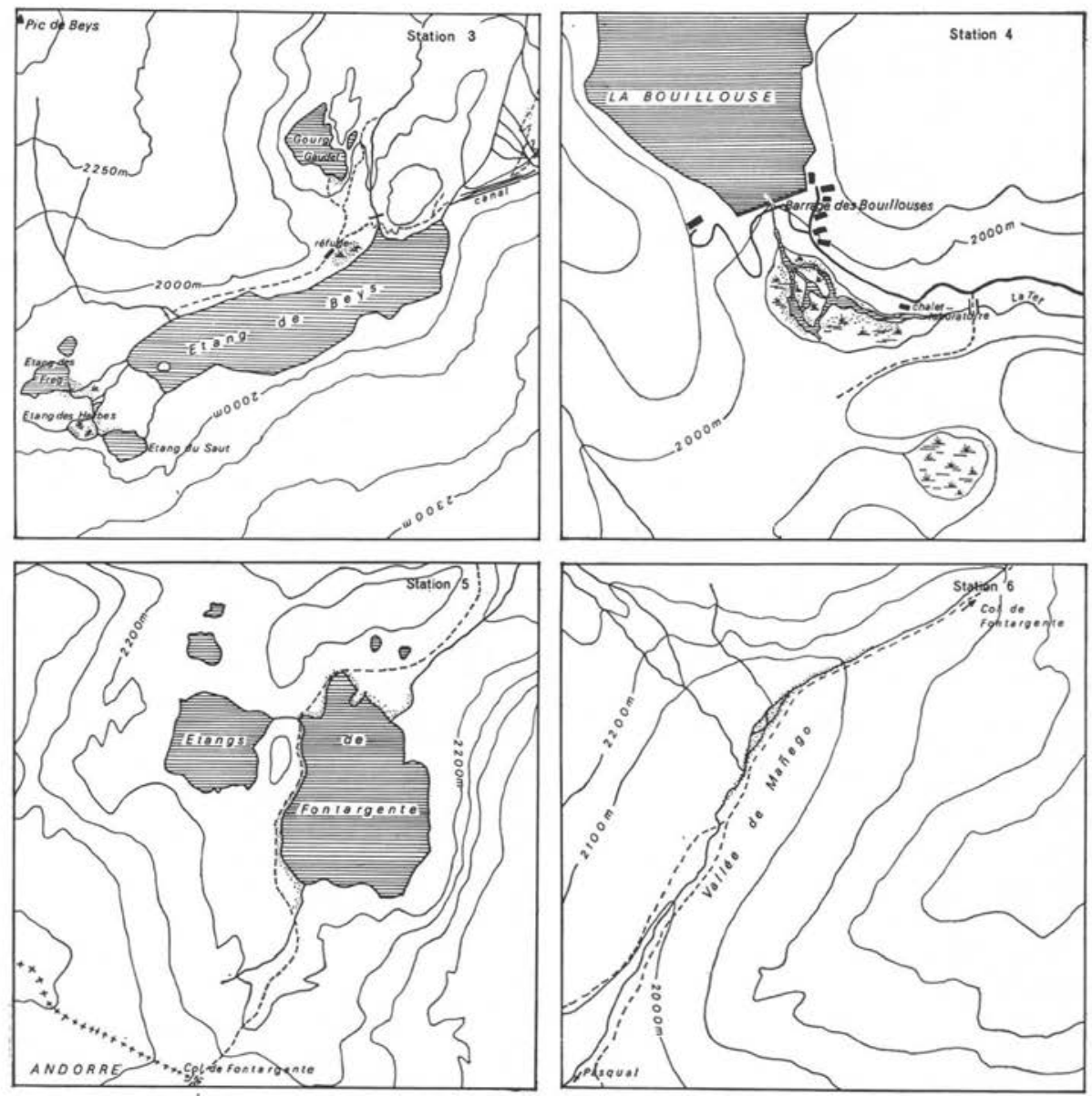

FIG. 2

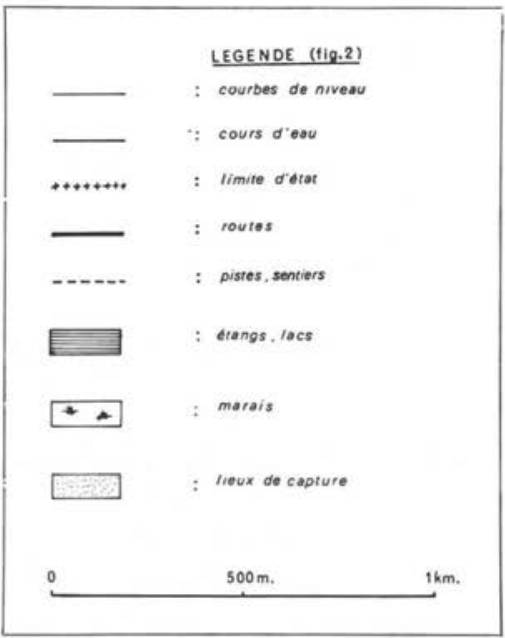

Annales de Parasitologie humaine et comparée (Paris), t. 46, $\mathrm{n}^{\circ} 6$ 

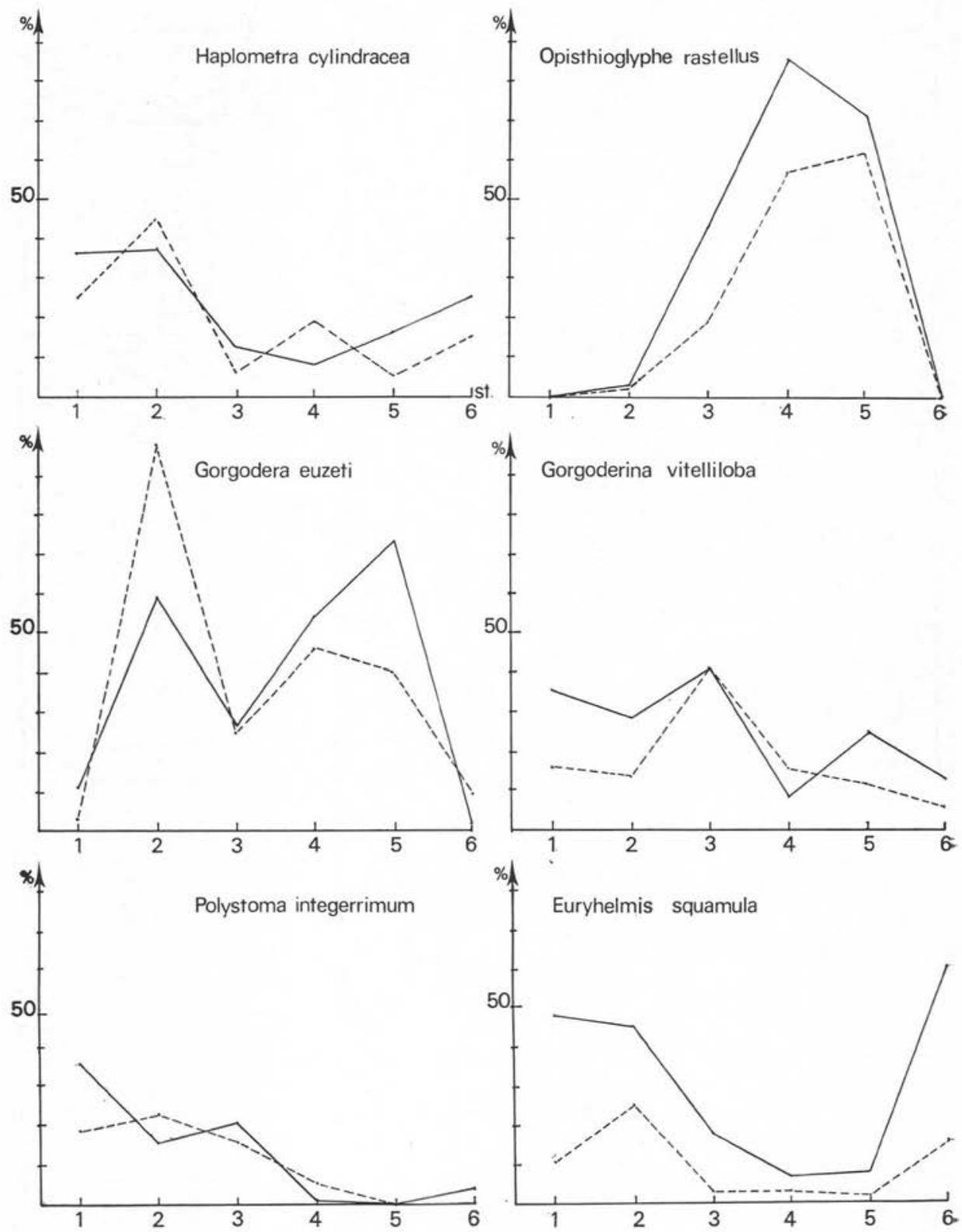

FIG. 3 

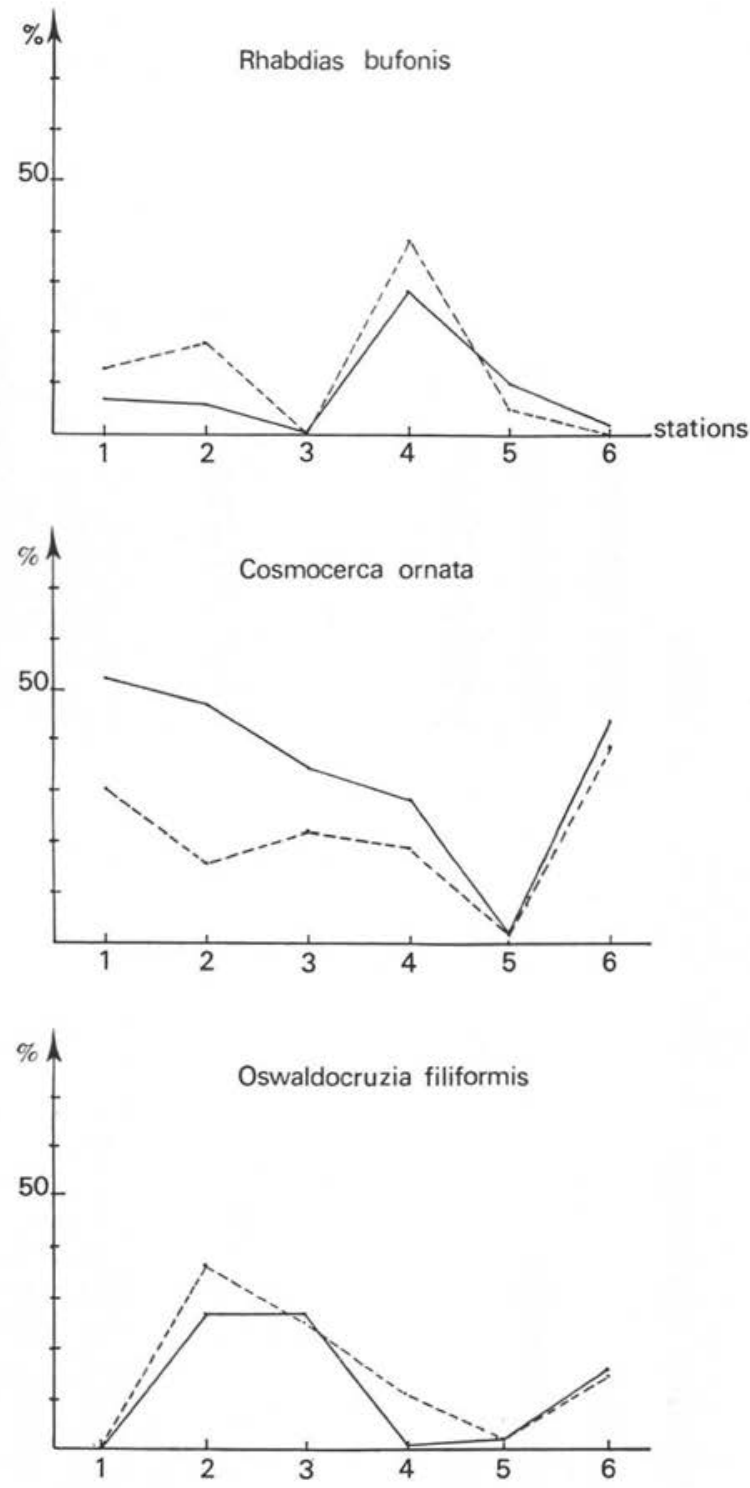

FIG. 4 

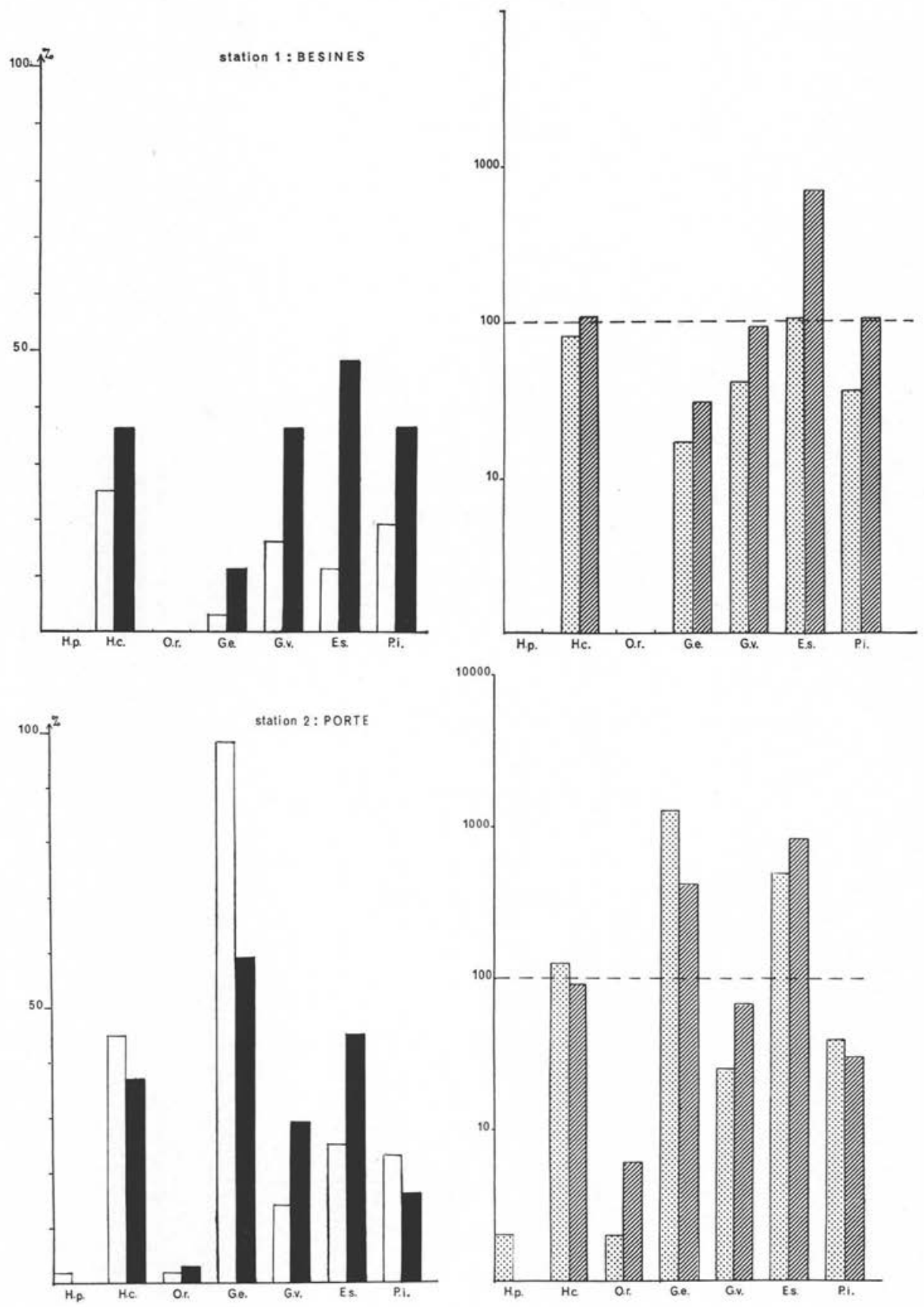

FIG. 5 


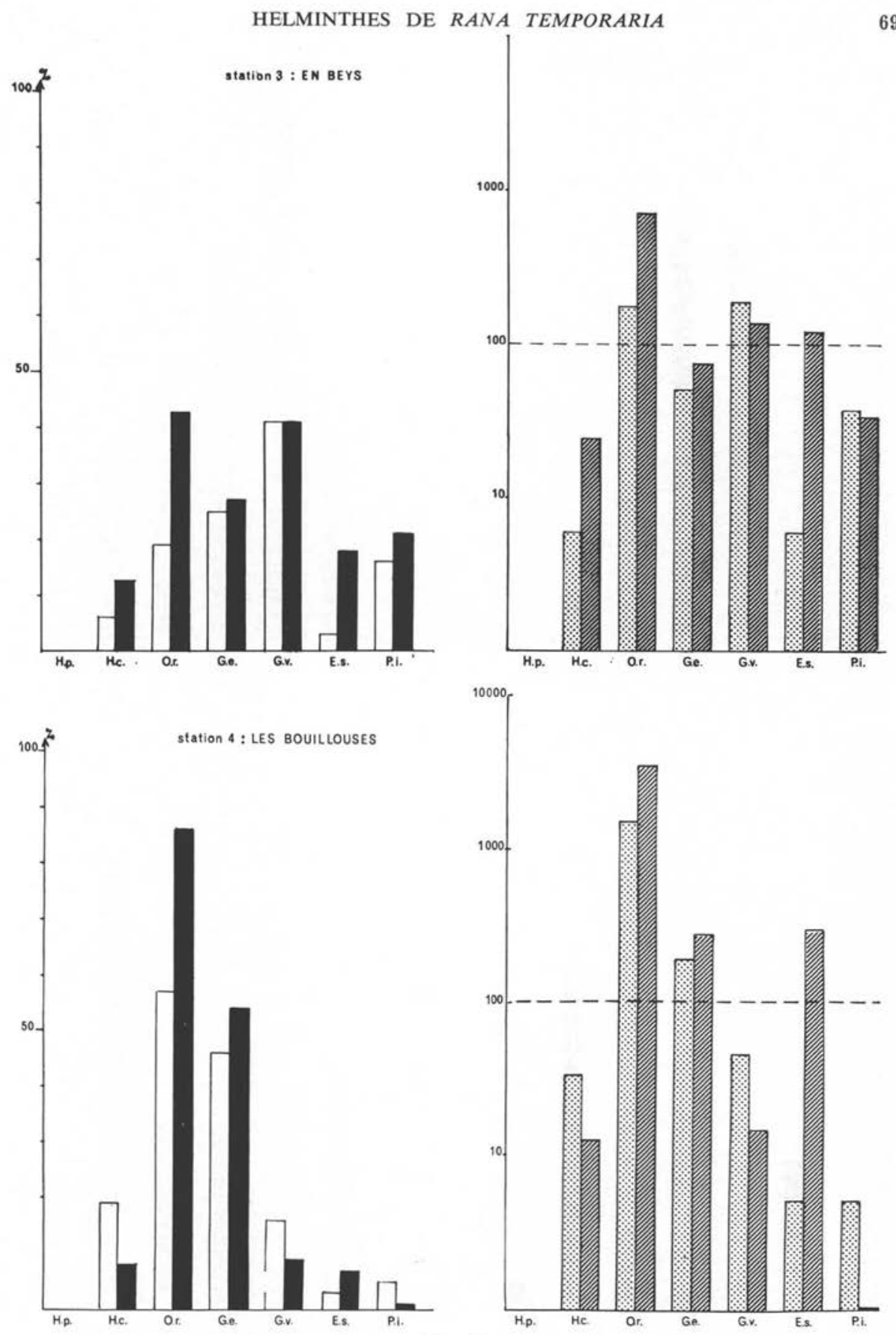

Fig. 6 

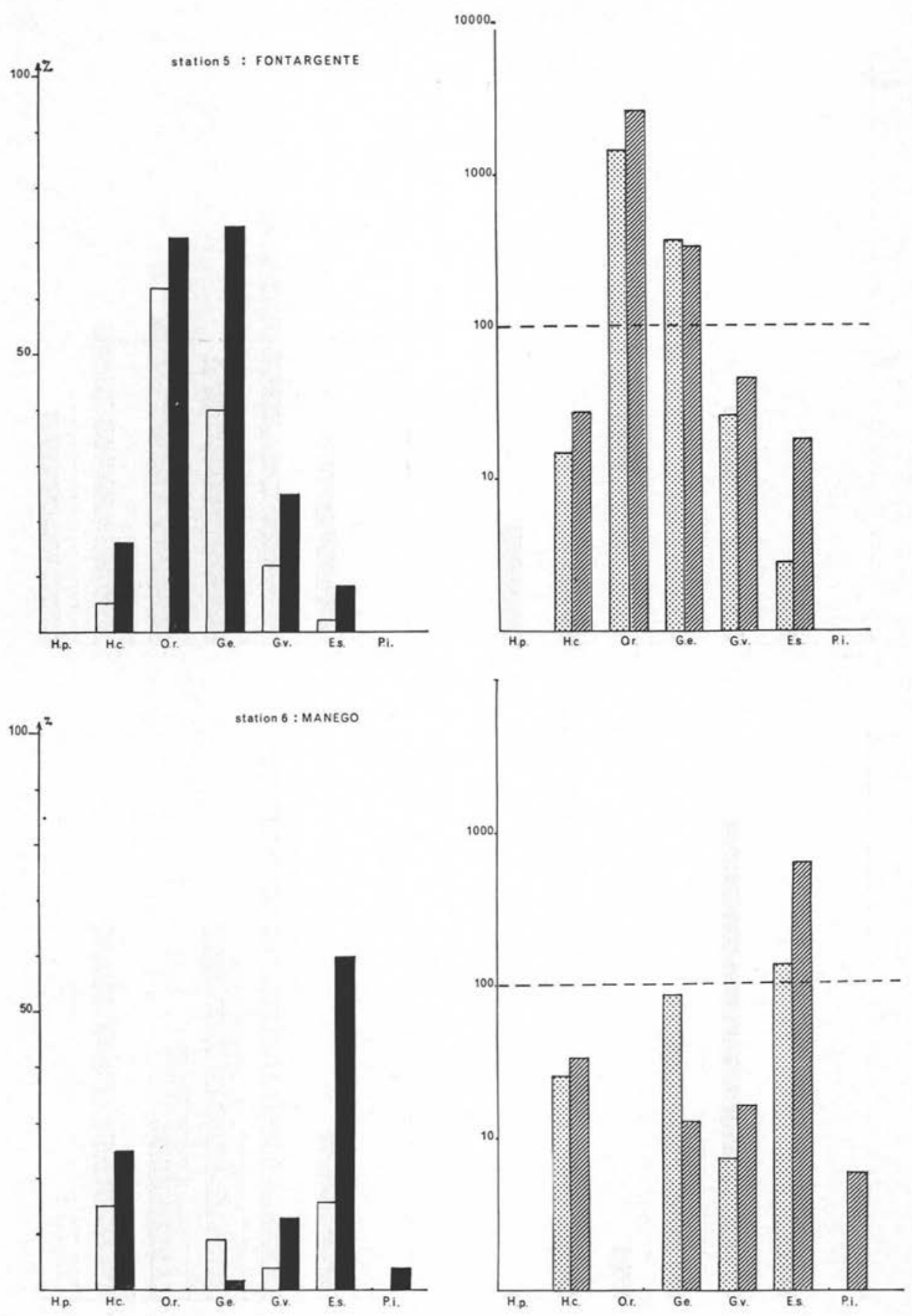

Fig. 7 

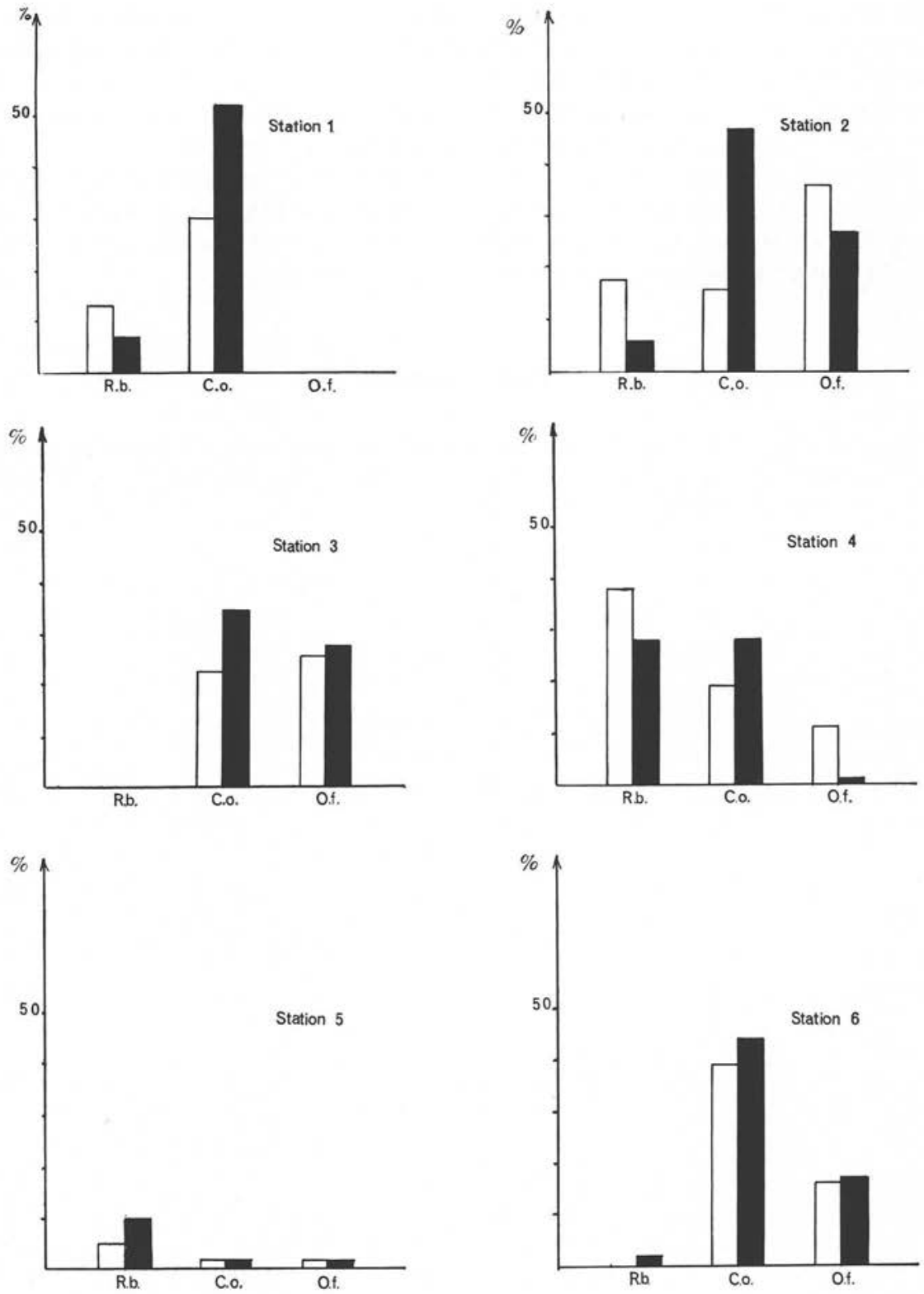

FIG. 8 
lités. Les seules variations observées peuvent s'expliquer par un simple mélange de populations de l'hôte entre deux cours d'eau voisins. On peut donc penser que les cartes biogéographiques publiées par Combes (1968) expriment davantage qu'une simple situation momentanée. Si des statistiques peuvent être ainsi réalisées dans l'avenir à des intervalles réguliers, peut-être mettra-t-on en évidence de lentes variations chorologiques du parasitisme, liées au réchauffement actuel du climat de cette région.

Sur le plan quantitatif, il va de soi qu'il serait vain de rechercher la signification des variations observées, car des statistiques annuelles, qui affaibliraient trop fortement les populations, ne sont pas faites. Il semble toutefois que c'est dans les milieux perturbés par l'homme qu'on note les fluctuations les plus importantes.

\section{Bibliographie}

Combes (C.), 1968. - Biologie, écologie des cycles et biogéographie de Digènes et Monogènes d'Amphibiens dans l'Est des Pyrénées. Mém. Mus. Nat. Hist. Nat. Paris. Nlle série, A, Zool. 51 : 1-196. 